\title{
Geometry-Based Stochastic Modeling for MIMO Channel in High-Speed Mobile Scenario
}

\author{
Binghao Chen and Zhangdui Zhong \\ State Key Laboratory of Rail Traffic Control and Safety, Beijing Jiaotong University, Beijing 100044, China \\ Correspondence should be addressed to Binghao Chen, bhchendo@gmail.com
}

Received 21 July 2012; Accepted 29 September 2012

Academic Editor: Ai Bo

Copyright $(\odot 2012$ B. Chen and Z. Zhong. This is an open access article distributed under the Creative Commons Attribution License, which permits unrestricted use, distribution, and reproduction in any medium, provided the original work is properly cited.

The geometry-based stochastic channel models are proposed in this paper for the terrain cutting, suburb, and urban scenarios in high-speed railway. First, the special scenarios in high-speed railway are described. And the channel models based on the geometry scenarios are introduced. Some channel parameters are based on measurement data. Then, the space-time correlation functions in analytical form are obtained in suburb and urban scenarios. Finally, the space correlation characteristics in three scenarios are compared.

\section{Introduction}

High-speed railway is playing an important role in the transportation of China. The wireless train control system is essential for the train management and safety. The train control system CTCS3 (Chinese Train Control System) based on GSM-R (Global System for Mobile Communication Railway) is applied to the train with the speed above $300 \mathrm{~km} / \mathrm{h}$. There is a rising demand to transmit large amount of data for the train safety and passenger service. So the wireless communication system will have an evolution from narrowband to wideband in the future. However, the frequency bandwidth allocated for the railway is limited. Therefore, the MIMO (Multiple-input Multiple-output) technique can be used in railway for it can enhance the channel capacity without additional bandwidth.

It is reported that the MIMO system performance strongly depends on the propagation environment. The scenarios in high-speed railway, such as viaduct and cutting, are special and different from those in commercial cellar communication. So the spatial channel should be studied well before the MIMO application. Some pathloss and channel characteristics in high-speed railway have been discussed in literatures. Wei et al. in [1] give an analysis of the pathloss in viaduct and plain scenarios based on the measurement data of Zhengzhou-Xi'an passenger dedicated line with the maximum speed of $340 \mathrm{~km} / \mathrm{h}$. Two modified pathloss models are proposed and compared with Hata model. The Ricean $K$ factors in viaduct and cutting scenarios are estimated in [2] based on the same measurement campaign. Lu et al. in [3] investigate the fitted pathloss model, shadow fading, and dynamic range of small-scale fading in viaduct and terrain cutting, which shows that the propagation environment of terrain cutting is worse than viaduct. The channel characteristics in high-speed railway for communication links based on Orthogonal Frequency Division Multiplexing (OFDM) are investigated in [4] using the ray-tracing tool. It seems that the researches mainly focus on the large-scale parameters and seldom refer to the small-scale channel characteristics and much less a MIMO channel model in high-speed railway. The geometry-based stochastic channel model (GSCM) is widely used in MIMO channel modeling [5]. The GSCM models can be classified into the regular-shaped GSCMs (RS-GSCMs) and irregularshaped GSCMs (IS-GSCMs) [6]. It depends on whether the effective scatterers distribute on a regular shape, for example, one-ring, two-ring, and ellipses or an irregular shape determined by the environment. RS-GSCMs are mathematically tractable and used for theoretical analysis. However, the IS-GSCM can easily handle the nonstationarity 
of mobile channel. Both the RS-GSCM and IS-GSCM models have been researched in Vehicle-to-Vehicle (VTV) scenario. In [7], a GSCM combining two-ring and one ellipse is proposed for mobile-to-mobile (M2M) scenario to study the space-time-frequency correlation, which is also the first model to take the vehicular traffic density into account. Then it is extended to a 3D M2M Ricean model [8]. And a wideband MIMO M2M model is proposed in [9]. It is assumed the scatterers are distributed in a set of concentric cylinders around the TX and RX. An IS-GSCM of VTV is proposed in [10], and the scatterers are distributed according to the environment geometry. A novel unified channel model for cooperative MIMO communication scenario is proposed in [11] with the ability to investigate the impact of the local scattering density on channel characteristics. Both the terminals in VTV and high-speed railway have a high speed, but the channel characteristics are different. In this paper, RS-GSCM and IS-GSCM are used to model the channels in terrain cutting, suburb, and urban scenarios.

The rest of this paper is organized as follows. The cutting scenario and the corresponding channel model are described in Section 2. The GSCMs in suburb and urban scenarios and the space-time correlation functions are introduced in Section 3. The simulations and compared results are shown in Section 4. Finally, Section 5 is the conclusion.

\section{The Cutting Channel Model}

2.1. Scenario Description. The terrain cutting scenario is shown in Figure 1. There are two slopes along the track. The height of the slope is about 13-14 $\mathrm{m}$. The angle of inclination is 35-40 degrees. And the distance between the two slopes is $12-13 \mathrm{~m}$. The slope is composed of concrete and stone with some grass on the surface.

2.2. Channel Model for Cutting Scenario. Based on the structure of cutting scenario, the channel model geometry is shown in Figure 2. The Base Stations (BSs) are built along the track. The distance between two BSs is about $3 \mathrm{~km}$. The BS is 10 to $30 \mathrm{~m}$ away from the track. The height of BS tower is about $30 \mathrm{~m}$. And the BS is built outside the cutting. The BS antennas face two directions along the track. Only the vertically polarized pattern field is considered. And the train antenna is $4.5 \mathrm{~m}$ high. The train is moving towards the BS. Only one BS is considered in simulation of this scenario.

The fitted pathloss model for cutting scenario is expressed as [3]:

$$
\mathrm{PL}_{\text {cutting }}(\mathrm{dB})=71.83+43 \log _{10}\left(\frac{d}{100}\right)+X_{\sigma} .
$$

The pathloss exponent $n=4.3 . d$ is the distance between the BS and the train. $X_{\sigma}$ is a zero-mean Gaussian random variable (in $\mathrm{dB}$ ) with standard deviation $3.5 \mathrm{~dB}$.

The scatterers are assumed to distribute uniformly on the surface of the two slopes with a density $\chi_{D I}$, stating the number of scatterers per meter. First, the scatterers can be generated in the $x-y$ plane. The $x$ coordinates of the scatterers follow the uniform distribution, that is, $x_{s} \sim U\left[x_{\min }, x_{\max }\right]$.

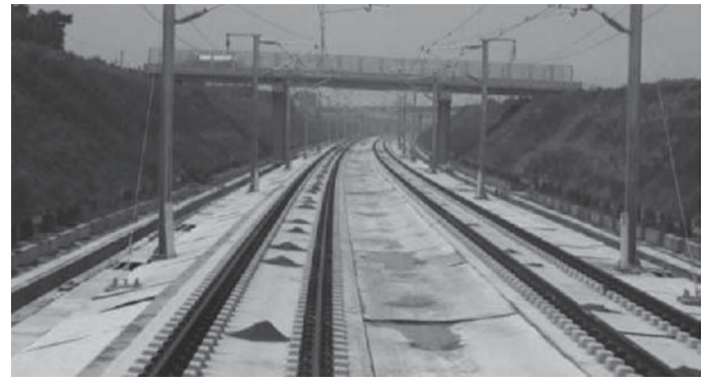

FIGURE 1: The cutting scenario in high-speed railway.

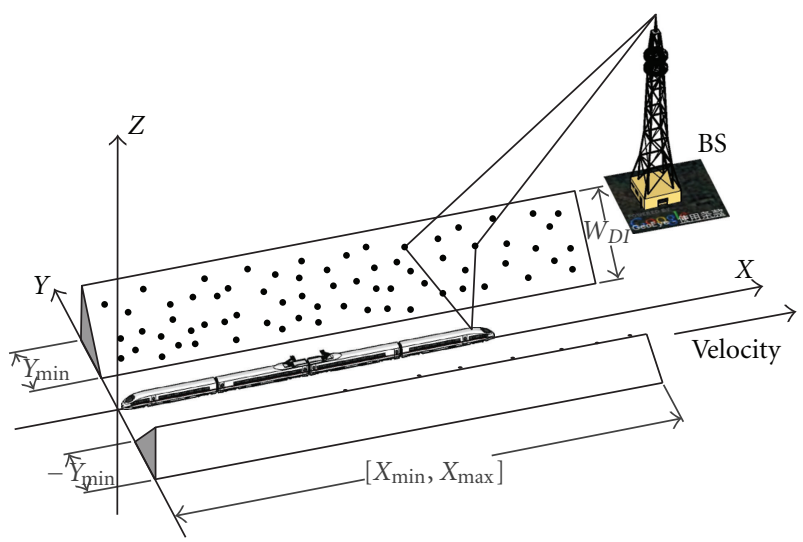

Figure 2: The channel model in terrain cutting.

And the $y$ coordinates in two semiaxis parts are $y_{s} \sim$ $U\left[y_{\min }, y_{\min }+W_{D I}\right]$ and $y_{s} \sim U\left[-y_{\min }-W_{D I},-y_{\min }\right]$, where $W_{D I}$ is the width of the slope area. Then the plane can be rotated to the angle of inclination to form the cutting slope.

The complex path gain of a diffuse scattering component in this GSCM is modeled as [10]:

$$
a_{s}=G_{0, D I}^{1 / 2} \cdot c_{s} \cdot\left(\frac{d_{\mathrm{ref}}}{d_{T \rightarrow r} \times d_{r \rightarrow R}}\right)^{n_{D I} / 2}
$$

where $c_{s}$ is the zero mean complex Gaussian variable. $n_{D I}$ and $G_{0, D I}$ are pathloss exponent and the reference power, respectively. It is assumed that $n_{D I}$ and $G_{0, D I}$ are the same for all diffuse scatterers. Only single-bounce path in this channel is considered. Since the slope is higher than the antenna on top of the train. Therefore, the power of paths from outside the cutting are neglected. In every time instant, the distance between the BS and the train is calculated. So are the distance from the BS to the scatterer and the distance from the scatterer to the train. The Angle-of-Arrival (AoA) and Angleof-Departure (AoD) are considered in three dimensions.

The channel is composed of Line-of-Sight (LOS) part and NLOS part, that is, the contribution from the diffuse scatterers in the environment. The three-dimensional 
double-directional channel impulse response for the diffuse components is expressed as:

$$
\begin{aligned}
& H_{s}=\sum_{m=1}^{M} a_{s} \cdot \exp \left(j \Phi_{m}\right) \cdot \exp \left(j d_{s} \frac{2 \pi}{\lambda} \bar{r}_{s} \cdot \bar{\Phi}_{m}\right) \\
& \cdot \exp \left(j d_{u} \frac{2 \pi}{\lambda} \bar{r}_{u} \cdot \bar{\Psi}_{m}\right) \cdot \exp \left(j 2 \pi v_{m} t\right),
\end{aligned}
$$

where $M$ is the total number of diffuse paths. $\Phi_{m}$ is the random phase for each path and $\Phi \sim U[0,2 \pi] . d_{s}$, and $d_{u}$ are the antenna spacing for the transmitter and receiver, respectively. $\lambda$ is the carrier wavelength. The scalar product is defined as [12]:

$$
\bar{r}_{s} \cdot \bar{\Phi}_{m}=x_{s} \cos \gamma_{m} \cos \phi_{m}+y_{s} \cos \gamma_{m} \sin \phi_{m}+z_{s} \sin \gamma_{m},
$$

where $\bar{r}_{s}$ is location vector of BS array element. $x_{s}, y_{s}, z_{s}$ are the coordinates of $\bar{r}_{s}$ to $x, y$, and $z$-axis, respectively. $\bar{\Phi}_{m}$ is departure angle unit vector. $\phi_{m}$ and $\gamma_{m}$ are the departure azimuth angle and departure elevation angle of the path $m$. And the scalar product $\bar{r}_{u} \cdot \bar{\Psi}_{m}$ is defined in the same way.

And $v_{m}$ is Doppler frequency for each path. The train is running along the track, so the Doppler Effect is considered in $x-y$ plane. $v_{m}$ is written as [12]

$$
\begin{aligned}
v_{m} & =\frac{\bar{v} \cdot \bar{\Psi}}{\lambda} \\
& =\frac{|v| \cos \theta_{v} \cos \gamma_{m} \cos \phi_{m}+|v| \sin \theta_{v} \cos \gamma_{m} \sin \phi_{m}}{\lambda},
\end{aligned}
$$

where $\theta_{v}$ is the mobile moving direction.

\section{Channel Models for Suburb and Urban}

The suburb and urban scenarios are kind of similar to those in commercial cell. So the RS-GSCM is used to model the channel in each scenario. Considering the narrowband system GSM-R used in high-speed railway, the spherical MIMO channel model is applied, which is appropriate for narrowband fading channel [13]. Also, the LOS and NLOS paths are considered separately.

The LOS case for $2 \times 2$ MIMO channel model is shown in Figure 3 . And the train is in the center of the scatterer sphere.

In the channel model, $D$ is the distance between BS and the train. $R$ is the radius of the sphere and related to the delay spread in this scenario. According to the real case, it is assumed $D \gg R$. $\delta_{p q}$ and $d_{l m}$ are the antenna spacing for BS and the train, with the antenna azimuth angles $a_{p q}$ and $\beta_{l m}$, respectively. For ease of description, the distance between two points $a$ and $b$ is written as $\xi_{a b}$. The AoA $\phi_{T}^{U}$ and elevation angle of LOS path in train side can be obtained through simple geometrical calculation.

In NLOS case, the scatterers are located in a spherical surface around the train. It is assumed that only one-bounce scattering occurs. The scatterers locations are described by two random variables, that is, the azimuth AoA $\phi_{i}^{U}$ and the elevation angle from scatterers $\beta$.
The von Mises distribution [14] is used to describe the azimuth angle $\phi_{i}^{U}$. The PDF (Probability Density Function) is written as

$$
f\left(\phi^{U}\right)=\frac{\exp \left[\kappa \cos \left(\phi^{U}-\mu\right)\right]}{2 \pi I_{0}(\kappa)}, \quad \phi^{U} \in[-\pi, \pi),
$$

where $\mu \in[-\pi, \pi)$ is the mean angle of the paths from the scatterers. The parameter $\kappa$ controls the spread around the mean angle. $I_{0}(\cdot)$ is the zero-order-modified Bessel function of the first kind.

The distribution of elevation angle from scatterers $\beta$ is defined as [15]

$$
f(\beta)= \begin{cases}\frac{\pi}{4|\beta|} \cos \left(\frac{\pi \beta}{2 \beta_{m}}\right), & |\beta| \leq\left|\beta_{m}\right| \leq \frac{\pi}{2} \\ 0, & \text { otherwise, }\end{cases}
$$

where $\beta_{m}$ is the maximum elevation angle.

According to the definition of correlation function in [14], the space-time cross correlation between two arbitrary communication links $h_{l p}(t)$ and $h_{m q}(t)$ is expressed as

$$
\rho_{l p, m q}(\tau, t)=\frac{E\left[h_{l p}(t) h_{m q}^{*}(t+\tau)\right]}{\sqrt{E\left[\left|h_{l p}(t)\right|^{2}\right] \cdot E\left[\left|h_{m q}(t)\right|^{2}\right]}} .
$$

By referring to the assumptions above and skipping some geometrical and algebra manipulations, the LOS and NLOS space-time correlation functions can be written as:

$$
\begin{aligned}
& \rho_{l p, m q}^{\operatorname{LOS}}(\tau)= \frac{K}{K+1} \\
& \times \exp \left[j c_{p q} \frac{\cos \left(a_{p q}\right)}{\cos \beta}+j b_{l m} \frac{\cos \left(\phi^{U}-\beta_{l m}\right)}{\cos \beta}\right. \\
&\left.-j a \cos \left(\phi^{U}-\gamma\right)\right] \\
& \rho_{l p, m q}^{\mathrm{DIF}}(\tau)=\frac{1}{K+1} \cdot \frac{\pi}{4 I_{0}(\kappa)\left|\beta_{m}\right|} \cdot \exp \left[\frac{j c_{p q} \cos \left(a_{p q}\right)}{\cos \beta_{T}}\right] \\
& \cdot \int_{-\beta_{m}}^{\beta_{m}} \cos \frac{\pi\left(\beta-\beta_{T}\right)}{2 \beta_{m}} \\
& \cdot I_{0}\left(\left\{\mathcal{K}^{2}-a^{2}-\frac{b_{l m}^{2}}{\cos \beta}-\frac{c_{p q}^{2} \Delta^{2} \sin ^{2} a_{p q} \cos { }^{2} \beta}{\cos \beta_{T}}\right.\right. \\
&+\frac{2 a b_{l m} \cos \left(\beta_{l m}-\gamma\right)}{\cos \beta}+\frac{2 c_{p q} \Delta \sin a_{p q} \cos \beta}{\cos \beta_{T}} \\
& \quad \cdot\left[a \sin \gamma-b_{l m} \frac{\sin \beta_{l m}}{\cos \beta}\right] \\
& \quad-j 2 \kappa\left[a \cos (\mu-\gamma)-\frac{b_{l m}}{\cos \beta} \cdot \cos \left(\mu-\beta_{l m}\right)\right. \\
&\left.\left.\left.\quad-\frac{c_{p q} \Delta \sin \left(a_{p q}\right) \sin \mu \cdot \cos \beta}{\cos \beta_{T}}\right]\right\}\right) d \beta
\end{aligned}
$$




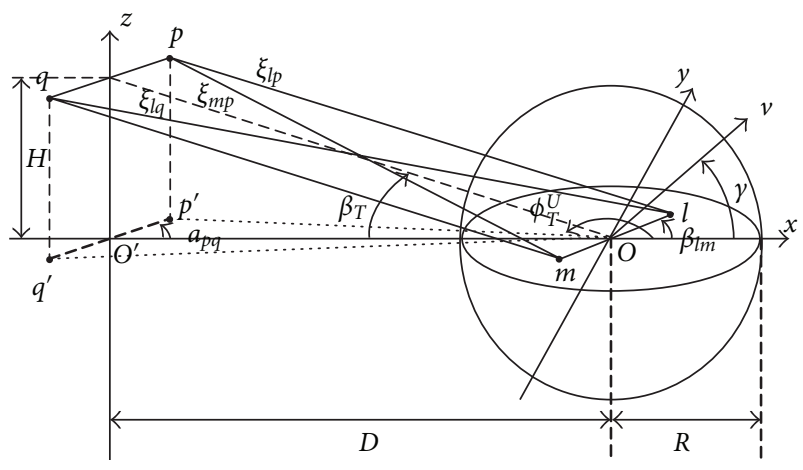

FIgUre 3: The $2 \times 2$ MIMO channel model with LOS path.

where $a=2 \pi f_{D} \tau, b_{l m}=2 \pi d_{l m} / \lambda$, and $c_{p q}=2 \pi \delta_{p q} / \lambda . K$ is the Ricean factor, defined as the ratio of the LOS component power to the diffuse components power. $f_{D}$ is the maximum Doppler frequency shift.

Finally, the total space-time cross correlation function $\rho_{l p, m q}$ is obtained by combining the (9):

$$
\rho_{l p, m q}(\tau)=\rho_{l p, m q}^{\mathrm{LOS}}(\tau)+\rho_{l p, m q}^{\mathrm{DIF}}(\tau) .
$$

\section{Simulation Results}

4.1. Simulation of Cutting Channel Model. Some channel parameters are given as follows.

(i) The carrier frequency in high-speed railway is $900 \mathrm{MHz}$. So the carrier wavelength is $0.33 \mathrm{~m}$. The velocity of the train is $360 \mathrm{~km} / \mathrm{h}$, equal to $100 \mathrm{~m} / \mathrm{s}$.

(ii) Both the BS and the train have two antenna elements. So it is a $2 \times 2 \mathrm{MIMO}$ link. And the broadside of antenna arrays in BS and train is along the track.

(iii) The scatterers are generated uniformly on the surface of two slopes with the density $\chi_{D I}=2$ per meter. The pathloss exponent $n_{D I}=3$ and the reference power $G_{0, D I}=23[9]$.

(iv) The Ricean $K$ factor in terrain cutting follows the log-normal distribution with the mean $0.94 \mathrm{~dB}$ and standard deviation $4.18 \mathrm{~dB}[2]$.

The model is generated in a deterministic approach. So the complex correlation coefficient in [10] is used. The correlation between two complex random variables $u$ and $v$ is defined as

$$
\rho=\frac{E\left[u v^{*}\right]-E[u] E\left[v^{*}\right]}{\sqrt{\left(E\left[|u|^{2}\right]-|E[u]|^{2}\right)\left(E\left[|v|^{2}\right]-|E[v]|^{2}\right)}},
$$

where $(\cdot)^{*}$ is complex conjugation. The coefficient $\rho$ denotes the space correlation between every two subchannels. The CDF (Cumulative Distribution Function) curves for different antenna spacing combinations are obtained.

According to the actual space limitation on top of the train, four combinations of BS and the train antenna spacing

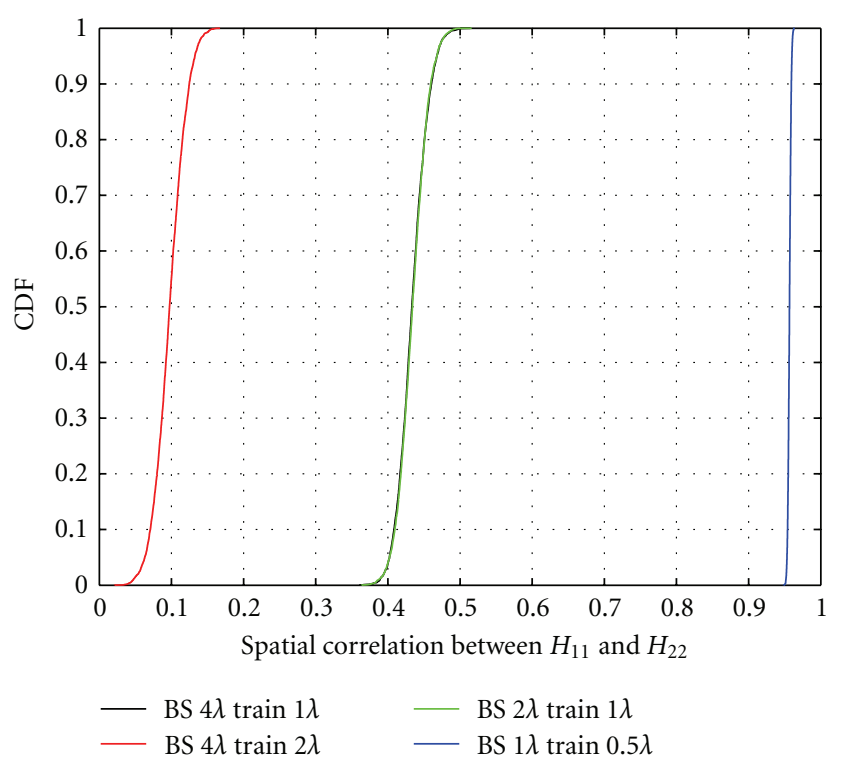

FIGURE 4: The space correlation in terrain cutting.

are chosen in the simulation. The correlation between the subchannels $H_{11}$ and $H_{22}$ is given in Figure 4. The curves of BS $4 \lambda$, the train $\lambda$ case and BS $2 \lambda$, the train $\lambda$ case are almost the same. It is shown that the antenna spacing of BS has little influence on the space correlation in railway scenario. However, when BS antenna spacing is fixed, as the train antenna spacing increases, the space correlation will change significantly.

4.2. Simulation of Suburb and Urban Channel Models. The space-time correlation function is obtained using (10). The parameters of the model are set as: $K$ (in $\mathrm{dB})$ $N(6.2,2)$ [2], $D=1000 \mathrm{~m}, R=10 \mathrm{~m}, H=30 \mathrm{~m}, \tau=0, a_{p q}=$ $90^{\circ}, \beta_{l m}=90^{\circ}$, the train moving direction $\gamma=180^{\circ}$, the velocity of the train $v=360 \mathrm{~km} / \mathrm{h}=100 \mathrm{~m} / \mathrm{s}, f_{D}=300 \mathrm{~Hz}$. The scatterers in suburb scenario of high-speed railway are limited. Therefore, the azimuth angle distribution is more concentrated [14]. On the contrary, the scatterers in urban scenario are much richer and the AoA spread is large. So the control parameter $\kappa$ for the azimuth angle spread is set as $\kappa=3$ and $\kappa=0$ for suburb and urban scenarios, respectively.

Figures 5 and 6 show the effect of antenna spacing on the space correlation under the two scenarios. The $x$ - and $y$-axis denote the antenna spacing for the train and BS. For $\kappa=0$, that is, the isotropic scattering, the minimum space correlation is lower than the correlation when $\kappa=3$. It is clear that the space correlation fluctuation is decreasing when $\kappa$ increases and the scattering components are getting more centralized. Therefore, the space correlation in suburb scenario is higher than that in urban area of high-speed railway under the same antenna spacing.

4.3. Space Correlations in Cutting, Suburb, and Urban. The channel modeling approach for terrain cutting is different from those in suburb and urban. So the space correlations 


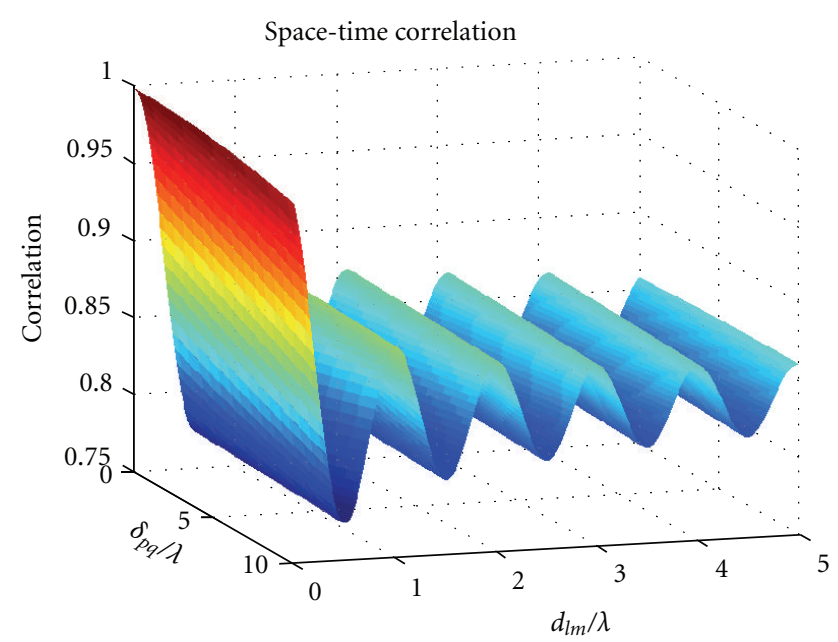

FIgURE 5: The space correlation when $\kappa=0$.

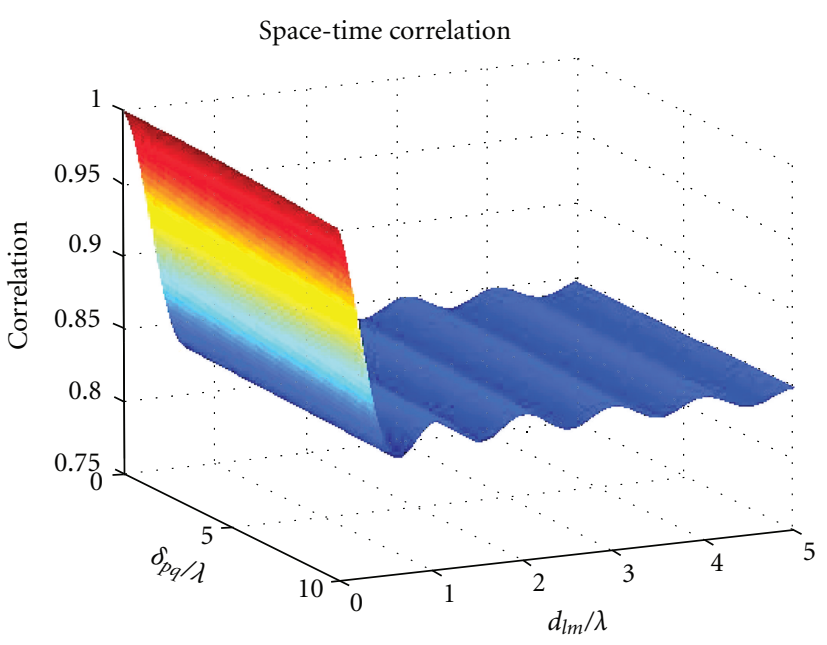

FIgURE 6: The space correlation when $\kappa=3$.

in these three scenarios are compared by the means of CDF curves, which are shown in Figure 7.

In Figure 7, two antenna spacing combinations are given. When BS spacing is $1 \lambda$ and the train spacing is $0.5 \lambda$, the space correlation is higher than those in suburb and urban. When BS spacing is changed into $2 \lambda$, the train spacing becomes $1 \lambda$, the space correlation changes more significantly than the other two scenarios. The reason is that the Ricean $K$ factor in terrain cutting is much lower and has a larger change than the other two scenarios. For the cutting slope is high, there are even bridges across the cutting to block the LOS path which can be seen in Figure 1. Therefore, the diffuse components power is relatively high. So a low correlation can be obtained when the antenna spacing increases. In general, the space correlation dynamic range in terrain cutting is larger than those in the other two scenarios.

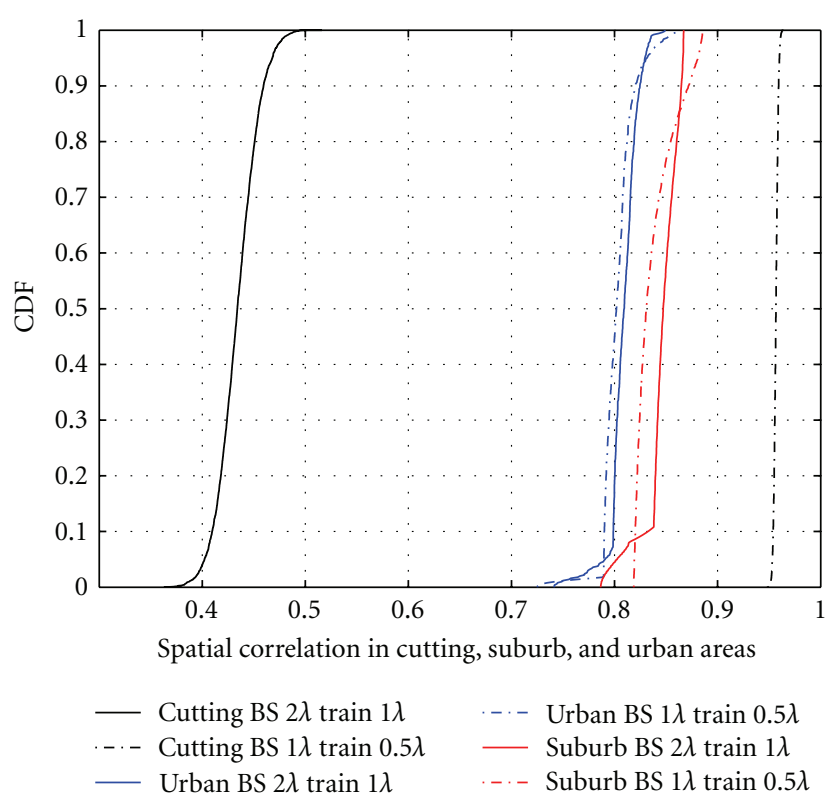

Figure 7: The CDF curves for the three scenarios.

\section{Conclusions}

In this paper, the MIMO channel modeling in high-speed railway is discussed including cutting, suburb, and urban scenarios. The IS-GSCM in cutting and RS-GSCM in suburb and urban scenarios are proposed. With the channel models, the space correlation coefficients in three scenarios are obtained. By comparing the simulation results, it shows that the train antenna spacing has a great impact on the space correlation. The space correlation in terrain cutting is more sensitive to the antenna spacing. And it has a greater dynamic range than the other two scenarios.

\section{Acknowledgments}

The project is supported by Program for New Century Excellent Talents in University under Grant NCET-09-0206; the National Natural Science Foundation of China under Grant 60830001; the Key Project of State Key Lab. of Rail Traffic Control and Safety under Grant RCS2011ZZ008; Program for Changjiang Scholars and Innovative Research Team in University under Grant no. IRT0949; the Project of State Key Lab. of Rail Traffic Control and Safety under Grant RCS2010ZT012; Beijing Natural Science Foundation (4112048), and "the Fundamental Research Funds for the Central Universities” under Grant nos. 2010JBZ008 and 2011YJS010.

\section{References}

[1] H. Wei, Z. Zhong, K. Guan, and B. Ai, "Path loss models in viaduct and plain scenarios of the high-speed railway," in Proceedings of the 5th International ICST Conference on Communications and Networking in China (ChinaCom '10), pp. 1-5, August 2010. 
[2] L. Gao, Z. Zhong, B. Ai, and L. Xiong, "Estimation of the Ricean K factor in the high speed railway scenarios," in Proceedings of the 5th International ICST Conference on Communications and Networking in China (ChinaCom '10), pp. 1-5, August 2010.

[3] J. Lu, G. Zhu, and C. Briso-Rodriguez, "Fading characteristics in the railway terrain cuttings," in Proceedings of the 73rd IEEE Vehicular Technology Conference (VTC Spring '11), pp. 1-5, 2011.

[4] S. Knörzer, M. A. Baldauf, T. Fügen, and W. Wiesbeck, "Channel modelling for an OFDM train communications system including different antenna types," in Proceedings of the IEEE 64th Vehicular Technology Conference (VTC '06 Fall), pp. 213-217, September 2006.

[5] P. Almers, E. Bonek, A. Burr et al., "Survey of channel and radio propagation models for wireless MIMO systems," Eurasip Journal on Wireless Communications and Networking, vol. 2007, Article ID 19070, 2007.

[6] C. X. Wang, X. Cheng, and D. Laurenson, "Vehicle-to-vehicle channel modeling and measurements: recent advances and future challenges," IEEE Communications Magazine, vol. 47, no. 11, pp. 96-103, 2009.

[7] X. Cheng, C. X. Wang, D. I. Laurenson, S. Salous, and A. V. Vasilakos, "An adaptive geometry-based stochastic model for non-isotropic MIMO mobile-to-mobile channels," IEEE Transactions on Wireless Communications, vol. 8, no. 9, pp. 4824-4835, 2009.

[8] X. Cheng, C. X. Wang, Y. Yuan, D. I. Laurenson, and X. Ge, "A novel 3D regular-shaped geometry-based stochastic model for non-isotropic MIMO mobile-to-mobile channels," in Proceedings of the IEEE 72nd Vehicular Technology Conference Fall (VTC'10-Fall), Ottawa, Canada, September 2010.

[9] A. G. Zajić, G. L. Stüber, T. G. Pratt, and S. T. Nguyen, "Wideband MIMO mobile-to-mobile channels: geometrybased statistical modeling with experimental verification," IEEE Transactions on Vehicular Technology, vol. 58, no. 2, pp. 517-534, 2009.

[10] J. Karedal, F. Tufvesson, N. Czink et al., "A geometry-based stochastic MIMO model for vehicle-to-vehicle communications," IEEE Transactions on Wireless Communications, vol. 8, no. 7, pp. 3646-3657, 2009.

[11] X. Cheng, C. X. Wang, H. Wang et al., "Cooperative MIMO channel modeling and multi-link spatial correlation properties," IEEE Journal on Selected Areas in Communications, vol. 30, no. 2, pp. 388-396, 2012.

[12] P. Kyosti, J. Meinila, L. Hentila et al., "WINNER II channel models, IST,” Tech. Rep. IST-4-027756 WINNER II D1.1.2 v1.2, 2007.

[13] J. Gong, J. F. Hayes, and M. R. Soleymani, "The effect of antenna physics on fading correlation and the capacity of multielement antenna systems," IEEE Transactions on Vehicular Technology, vol. 56, no. 4 I, pp. 1591-1599, 2007.

[14] A. Abdi and M. Kaveh, "A space-time correlation model for multielement antenna systems in mobile fading channels," IEEE Journal on Selected Areas in Communications, vol. 20, no. 3, pp. 550-560, 2002.

[15] A. A. Al-Kheir, K. A. Qaraqe, and M. S. Alouini, "On the space-time correlation of MIMO fading channels in $3 \mathrm{D}$ scattering models," in Proceedings of the 13th IEEE Symposium on Computers and Communications (ISCC '08), pp. 831-837, July 2008. 

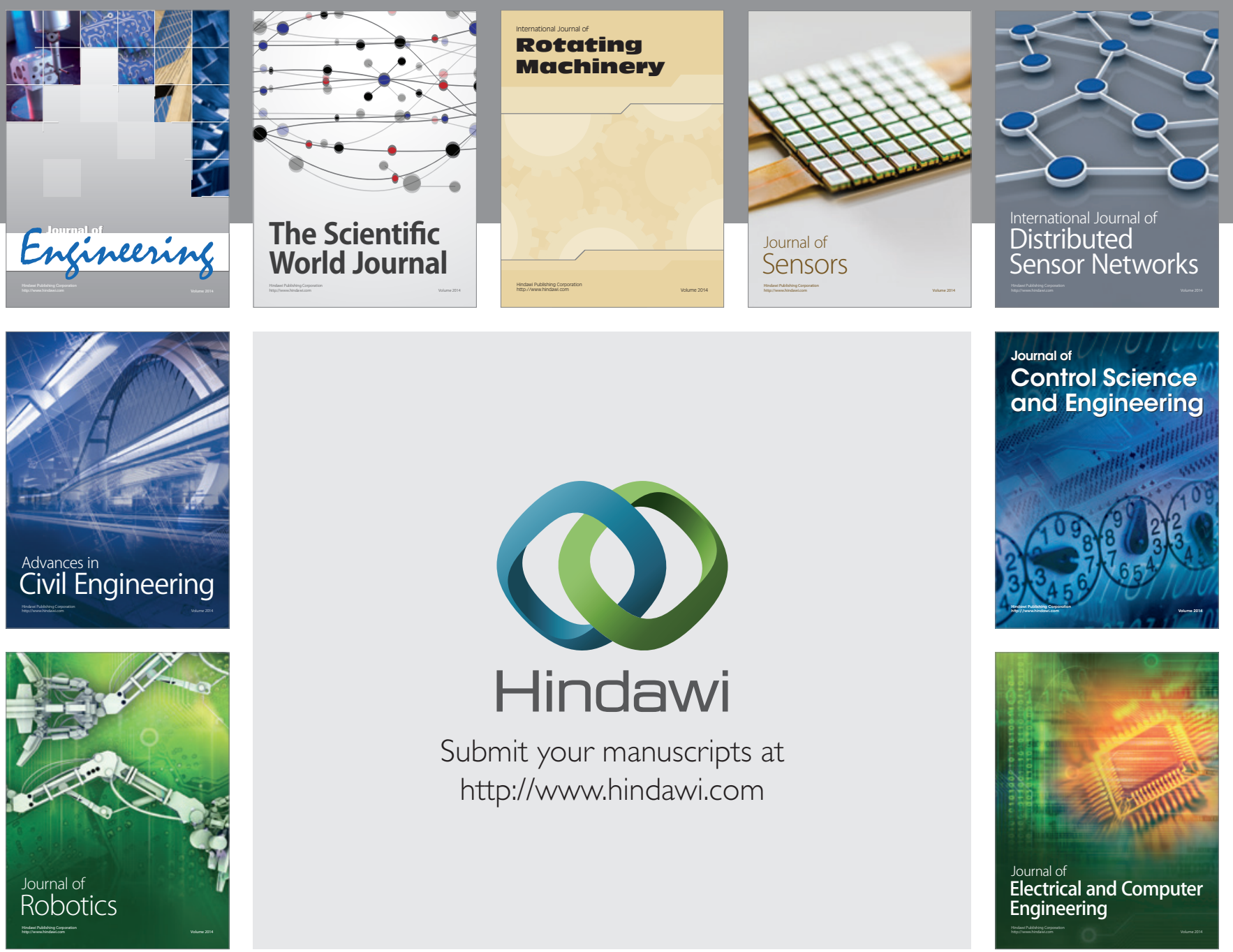

Submit your manuscripts at

http://www.hindawi.com
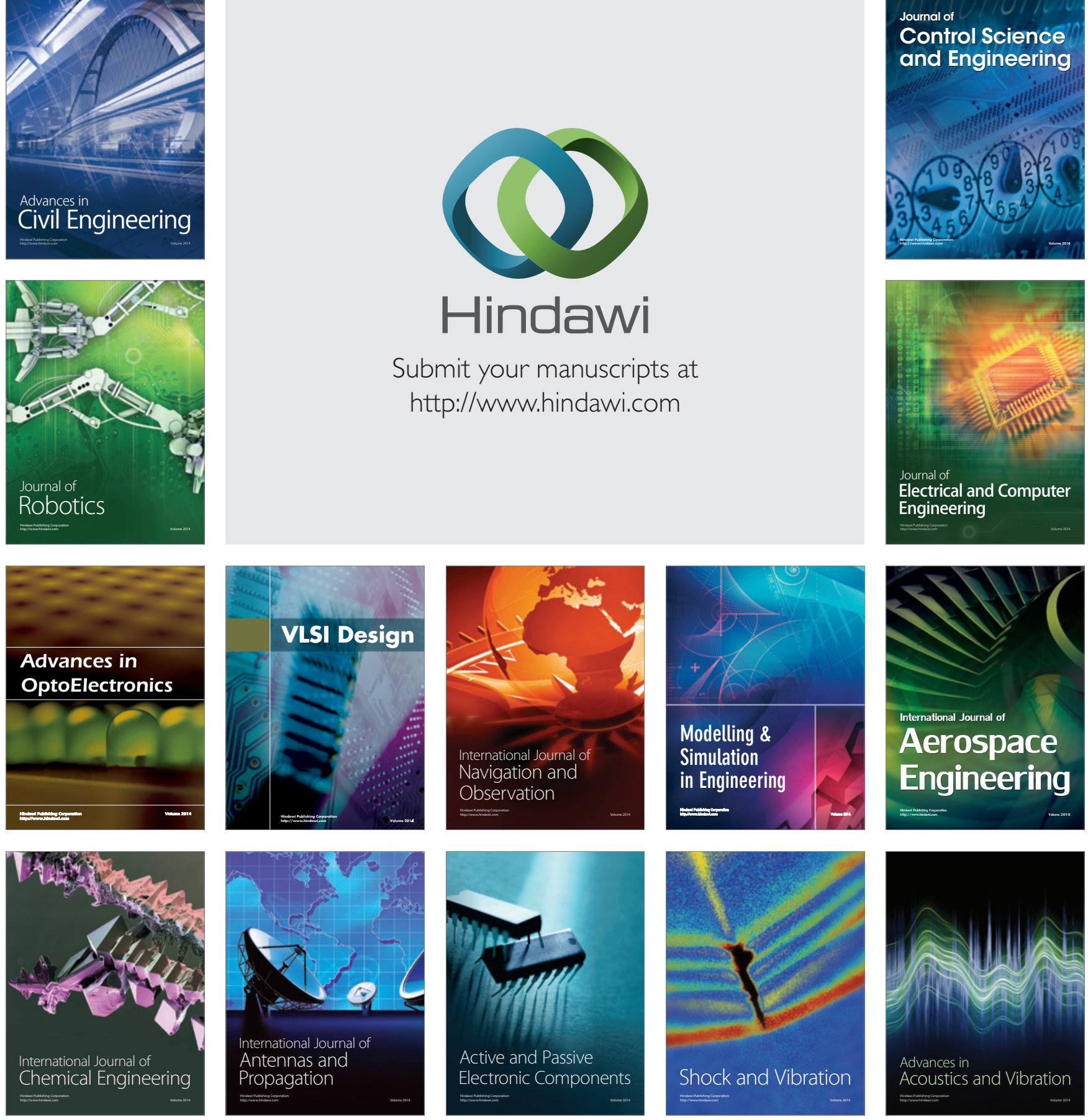